\title{
Поглощение излучения дальнего инфракрасного диапазона квантовыми точками Ge/Si
}

\author{
(C) А.Н. Софронов ${ }^{1}$, Р.М. Балагула ${ }^{1}$, Д.А. Фирсов ${ }^{1}$, Л.Е. Воробьев ${ }^{1}$, А.А. Тонких ${ }^{2}$, \\ А.А. Саркисян ${ }^{1,3}$, Д.Б. Айрапетян ${ }^{3}$, Л.С. Петросян ${ }^{3,4}$, Э.М. Казарян ${ }^{3}$ \\ ${ }^{1}$ Санкт-Петербургский политехнический университет Петра Великого, \\ 195251 Санкт-Петербург, Россия \\ ${ }^{2}$ OSRAM Opto Semiconductors, \\ Regensburg, Germany \\ ${ }^{3}$ Российско-Армянский университет, \\ Ереван, Армения \\ ${ }^{4}$ Университет Джексона, \\ США \\ E-mail: sofronov@rphf.spbstu.ru
}

(Получена 22 мая 2017 г. Принята к печати 31 мая 2017 г.)

Рассмотрены экспериментальные и теоретические результаты исследования оптического поглощения легированных структур с квантовыми точками $\mathrm{Ge} / \mathrm{Si}$ в дальнем инфракрасном диапазоне, соответствующем энергиям переходов дырок из основного в нижнее возбужденное состояние размерного квантования. В рамках адиабатического приближения построена аналитическая теория размерного квантования дырок в линзообразной квантовой точке с учетом парного кулоновского взаимодействия. Показано, что наличие взаимодействия не оказывает влияния на частоты нижних межуровневых резонансов, что является отражением реализации обобщенной теоремы Кона благодаря специфической геометрии квантовой точки. Экспериментальные и теоретические значения энергии переходов находятся в хорошем согласии.

DOI: 10.21883/FTP.2018.01.45320.8655

\section{1. Введение}

Интерес к структурам с квантовыми точками (КТ) $\mathrm{Ge} / \mathrm{Si}$ обусловлен в первую очередь, их совместимостью с существующей кремниевой технологией. В ряде работ [1] показано, что KT Ge/Si обладают как привлекательными люминесцентными свойствами, так и фотопроводимостью в области энергий кванта излучения, меньшей ширины запрещенной зоны кремния. Это открывает широкие возможности по использованию таких структур в качестве материалов активной элементной базы для оптических межсоединений в микроэлектронике [2]. В недавней работе [3] сообщается о наблюдении при комнатной температуре стимулированного излучения вблизи 1.3 мкм из частично аморфных германиевых КТ в кремниевой матрице внутри микродискового резонатора при оптической накачке.

Оптические свойства KT Ge/Si в средней инфракрасной (ИК) области также представляют существенный интерес в связи с потенциальными возможностями использования их для фотоприемников на этот диапазон $[4,5]$. В этом спектральном диапазоне привлекают внимание внутризонные оптические явления, связанные с переходами дырок между размерно-квантованными состояниями валентной зоны КТ, на которую приходится бо́льшая часть разности в ширине запрещенной зоны германия и кремния. При этом интерфейс квантовой точки формирует сильное квантовое ограничение для дырок внутри КТ, тогда как электроны могут быть только слабо локализованы на кремниевой части интерфейса за счет изгибов зоны проводимости, вызванных наличием остаточных упругих напряжений [6].
Несмотря на большое количество исследований квантовых точек на основе этой системы материалов, изучению оптических свойств КТ $\mathrm{Ge} / \mathrm{Si}$ в дальней ИК спектральной области уделялось мало внимания. При этом в данной спектральной области находятся энергии оптических переходов дырок из основного состояния в нижние возбужденные состояния КТ, изучение которых позволяет исследовать некоторые особенности, связанные с распределением дырок по состояниям КТ с неоднородным уширением, что может быть важным при анализе работы оптоэлектронных приборов.

В данной работе мы приводим результаты экспериментального исследования спектров оптического поглощения легированных структур с квантовыми точками $\mathrm{Ge} / \mathrm{Si}$ в дальней ИК области, а также аналитическую теорию межуровневого поглощения света на нижнем внутризонном резонансе с учетом кулоновского взаимодействия дырок в квантовой точке.

\section{2. Методика эксперимента}

В эксперименте исследовались структуры с самоорганизованными квантовыми точками $\mathrm{GeSi} / \mathrm{Si}$, выращенные методом молекулярно-пучковой эпитаксии на высокоомных кремниевых подложках (001). Структура состояла из 10 слоев квантовых точек, полученных осаждением 7 монослоев германия при температуре $600^{\circ} \mathrm{C}$ со скоростью 0.14 монослоя в секунду. Соседние слои с квантовыми точками отделялись слоями собственного кремния толщиной 15 нм. Рост проводился с использованием сурьмы в качестве сурфактанта, что позволило 
увеличить плотность квантовых точек. Влияние сурьмы детально описано в $[7,8]$, в частности показано, что в процессе роста сурьма остается на поверхности структуры и ее добавка не приводит к непреднамеренному $n$-легированию. Содержание германия в материале квантовых точек, выращенных в таких условиях, составляет $60-65 \%$ [9].

Структурные параметры квантовых точек исследовались с помощью провечивающей электронной и атомносиловой микроскопии [7-9]. Плотность квантовых точек оценивается как $2 \cdot 10^{11} \mathrm{~cm}^{-2}$. Геометрия квантовых точек показывает достаточно большое отношение характерных размеров в плоскости слоев и вдоль оси роста. Так, средняя высота составляет 2.7 нм с разбросом в 0.25 нм, характерный размер в плоскости слоя - 14 нм с разбросом в 4 нм.

Структуры селективно легировались бором в $\delta$-слое, расположенном на расстоянии 5 нм под каждым слоем точек. Поверхностная концентрация бора в разных структурах в $\delta$-слое составляла $4 \cdot 10^{11}, 8 \cdot 10^{11}$ и $12 \cdot 10^{11} \mathrm{~cm}^{-2}$, обеспечивая номинальный уровень легирования 2, 4 и 6 дырок на точку соответственно. Кроме того, структура, выращенная в таких же условиях без преднамеренного легирования, использовалась в качестве референтной.

Ранее были исследованы оптические свойства этих структур в среднем ИК диапазоне и показано, что основное состояние КТ лежит на 300 мэВ ниже валентной зоны кремния [10].

Из структур изготавливались образцы для оптических исследований в многопроходной геометрии, обеспечивающей увеличение длины оптического пути для детектирования слабого изменения оптического пропускания. За счет 5 полных внутренних отражений от одной поверхности структуры свет проходил слои с квантовыми точками 10 раз.

Оптическое пропускание квантовых точек исследовалось на экспериментальной установке на базе фурьеспектрометра Bruker Vertex 80v. Образцы с КТ монтировались в заливной оптический азотный криостат с входным и выходным окнами из полиэтилена. Криостат устанавливался в камеру образца спектрометра и позволял стабилизировать температуру образцов от 80 до $300 \mathrm{~K}$. Излучение глобара, прошедшее через образец, детектировалось кремниевым болометрическим фотоприемником, охлаждаемым жидким гелием. Вся оптическая система вместе с установленным светоделителем из майлара и проходной оптикой обеспечивала возможность измерений оптического пропускания в спектральном диапазоне $100-500 \mathrm{~cm}^{-1}$. Спектральное разрешение составляло $16 \mathrm{~cm}^{-1}$. Спектры пропускания исследовались в неполяризованном свете.

\section{3. Экспериментальные результаты}

На рис. 1 представлены экспериментальные спектры безразмерного коэффициента поглощения $\alpha L$ при трех

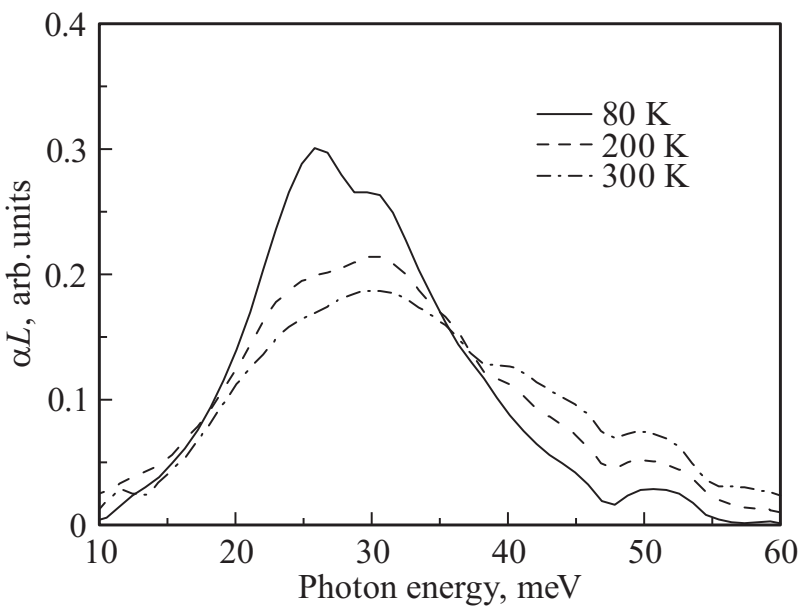

Рис. 1. Спектры поглощения структуры с КT $\mathrm{GeSi} / \mathrm{Si}$ с номинальным уровнем легирования 2 дырки на КТ при разных температурах.

температурах, определенные из спектров пропускания легированного образца с номинальным уровнем легирования 2 дырки на точку, нормированных на пропускание нелегированного образца.

Низкотемпературный спектр поглощения представляет собой широкий пик с центром вблизи энергии кванта падающего излучения 30 мэВ. Неоднородное уширение обусловлено разбросом геометрических размеров квантовых точек.

Нормировка экспериментальных данных на пропускание нелегированного образца позволяет утверждать, что наблюдаемое поглощение обусловлено переходами дырок из основного в одно из нижних возбужденных дырочных состояний квантовой точки. В отдельном эксперименте наблюдалось также фотоиндуцированное поглощение в том же спектральном диапазоне в нелегированных структурах с КТ $\mathrm{GeSi} / \mathrm{Si}$, обусловленное неравновесными дырками, возбужденными межзонной накачкой и захваченными в квантовые точки [10].

Увеличение температуры приводит к уширению спектра поглощения в коротковолновую область, что соответствует некоторому перераспределению дырок между квантовыми точками в ансамбле и заполнению точек меньшего размера.

Экспериментальные кривые поглощения для образцов с большим уровнем легирования имеют схожий характер. При этом спектральное положение пика поглощения остается неизменным.

Номинальный уровень легирования, обеспечивающий заполнение квантовых точек в среднем двумя дырками, приводит к наличию в ансамбле заряженных квантовых точек с зарядом больше заряда электрона $e$. Стоит отметить, что при низких температурах заполненными в ансамбле оказываются преимущественно КТ большого размера, как в силу меньшего значения энергии основного состояния размерного квантования дырок, 
так и в силу меньшей по величине добавки к нему энергии кулоновского взаимодействия дырок в КТ за счет большего „эффективного расстояния“ между взаимодействующими частицами.

Для корректного теоретического описания системы необходимо учитывать многочастичные взаимодействия. Рассмотрим для простоты вопрос об энергии первого межуровневого внутризонного оптического резонанса в одной КТ с несколькими взаимодействующими между собой дырками, пренебрегая наличием неоднородного уширения состояний ансамбля. Здесь следует особо отметить, что резонансные переходы в КТ, содержащих несколько частиц (дырок), могут иметь особый характер, обусловленный наличием эффективного ограничивающего параболического потенциала КТ. Как показано в работах [11-13], в таком случае в системе реализуется ситуация, когда, несмотря на наличие в КТ многочастичного газа, переходы носят одночастичный характер. Данное утверждение лежит в основе обобщенной теоремы Кона, согласно которой частота резонансных переходов в КТ с параболическим потенциалом ограничения не зависит от кулоновского взаимодействия между частицами и, следовательно, от числа частиц [11-13] (оригинальная теорема Кона была доказана для случая электронного газа в однородном магнитном поле [14]). В работах $[15,16]$ на основе адиабатического метода было показано, что в КТ с экстремальной геометрией сильно сплюснутого эллипсоида в плоскости слоя КТ реализуется двумерный параболический потенциал ограничения. Как следствие, выполняются условия для реализации обобщенной теоремы Кона.

Форма исследуемых KT Ge/Si может быть описана как сплюснутая квантовая линза, геометрия которой подобна геометрии сплюснутого эллипсоида. Таким образом, можно ожидать, что в рассматриваемой системе КТ $\mathrm{Ge} / \mathrm{Si}$ также могут иметь место переходы, характеризуемые обобщенной теоремой Кона.

\section{4. Теоретическая модель}

В качестве геометрической модели точки $\mathrm{Ge} / \mathrm{Si}$ pacсмотрим КТ в форме тонкой плоско-выпуклой сферической квантовой линзы (рис. 2) с непроницаемыми стенками. Кроме того, будем считать несущественным перемешивание состояний легких и тяжелых дырок и рассматривать только тяжелые дырки, а также пренебрежем наличием конечного разрыва валентной зоны на интерфейсе точки, поскольку он на порядок превышает энергии рассматриваемых переходов.

В указанном приближении ограничивающий потенциал, действующий на каждую из частиц, будет иметь следующий вид:

$$
U_{\text {conf }}(\rho, \varphi, z)= \begin{cases}0, & \text { inside } \\ \infty, & \text { outside }\end{cases}
$$

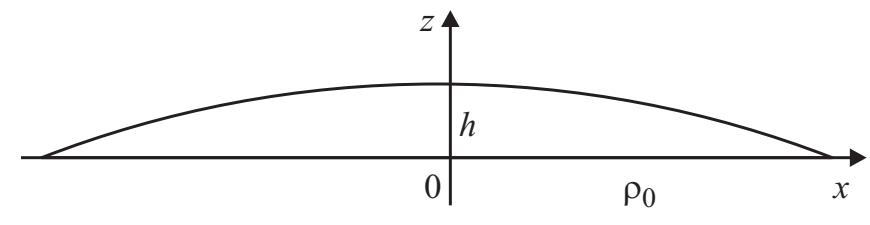

Рис. 2. Плоско-выпуклая линзообразная КТ.

В цилиндрических координатах одночастичное уравнение Шредингера запишется как

$$
\begin{array}{r}
-\frac{\hbar^{2}}{2 \mu}\left[\frac{1}{\rho} \frac{\partial}{\partial \rho}\left(\rho \frac{\partial}{\partial \rho}\right)+\frac{1}{\rho^{2}} \frac{\partial^{2}}{\partial \varphi^{2}}+\frac{\partial^{2}}{\partial z^{2}}\right] \Psi(\rho, \varphi, z) \\
+U_{\operatorname{conf}}(\rho, \varphi, z) \Psi(\rho, \varphi, z)=E \Psi(\rho, \varphi, z),
\end{array}
$$

где $\mu$ - эффективная масса тяжелой дырки (для твердого раствора $\mathrm{Ge}_{0.6} \mathrm{Si}_{0.4}$, формирующего материал КТ, значение $\mu=0.39 m_{0}$ получено линейной интерполяцией масс кремния и германия, $m_{0}-$ масса свободного электрона). Условие тонкости линзы имеет вид

$$
\rho_{0} \gg h
$$

где $h$ и $\rho_{0}$ - аксиальная и радиальная полуоси соответственно. С учетом условия (3) мы можем искать решение проблемы в рамках адиабатического приближения [17]. Геометрическая специфика КТ такова, что движение дырки вдоль оси $z$ происходит со значительно большей частотой, чем в перпендикулярной к ней плоскости. В таком случае волновую функцию дырки можно представить [15-18] в виде произведения

$$
\Psi(\rho, \varphi, z)=f(\rho) \chi(z(\rho))
$$

где $f(\boldsymbol{\rho})$-волновая функция, описывающая поведение дырки в плоскости, перпендикулярной оси $z, \chi(z(\rho))-$ волновая функция, описывающая движение вдоль оси $z$ и параметрически зависящая от координаты $\rho$ „медленной подсистемы“.

Для фиксированного значения $\rho$ дырка будет локализована в одномерной бесконечно глубокой яме вдоль оси $z$. При этом значение координаты $z$ будет меняться в пределах

$$
0 \leq z \leq \sqrt{R^{2}-\rho^{2}}+h-R,
$$

где $R$ - радиус кривизны поверхности линзы. Таким образом, при фиксированном значении радиальной координаты дырка будет совершать движение в одномерной непроницаемой яме шириной

$$
a(\rho)=\sqrt{R^{2}-\rho^{2}}+h-R
$$


Следовательно, для волновой функции и энергетического спектра аксиального движения можно записать

$$
\begin{gathered}
\chi_{n}(z(\rho))=\sqrt{\frac{2}{a(\rho)}} \sin \frac{\pi n}{a(\rho)} z, \\
E_{n}^{(z)}=\frac{\pi^{2} \hbar^{2} n^{2}}{2 \mu a^{2}(\rho)} \equiv U_{n}^{\mathrm{eff}}(\rho) .
\end{gathered}
$$

Заметим, что, согласно адиабатическому приближению, энергия $E_{n}^{\mathrm{eff}}(\rho)$ играет роль эффективной потенциальной энергии $U_{n}^{\mathrm{eff}}(\rho)$ для движения в плоскости сечения линзы [15-18]. Так как число дырок $N$ мало и размерное квантование в направлении оси $z$ является достаточно сильным, большинство дырок будет локализовано в геометрическом центре КТ. В таком случае можно считать, что имеет место соотношение $\rho \ll \rho_{0}$. Разлагая выражение для эффективной потенциальной энергии в ряд Тейлора по малому параметру $\rho / \rho_{0}$, находим:

$$
U_{n}^{\mathrm{eff}}(\rho)=\frac{\pi^{2} \hbar^{2} n^{2}}{2 \mu h^{2}}\left(1+\frac{\rho^{2}}{h R}\right)=\frac{\pi^{2} \hbar^{2} n^{2}}{2 \mu h^{2}}+\frac{\mu \Omega_{n}^{2} \rho^{2}}{2},
$$

где $\Omega_{n}^{2}$ имеет следующий вид:

$$
\Omega_{n}^{2}=\frac{\pi^{2} \hbar^{2}}{\mu^{2} h^{3} R} n^{2}
$$

Если теперь рассматривать газ с парным кулоновским взаимодействием

$$
V_{\text {int }}\left(\mathbf{r}_{1}, \ldots, \mathbf{r}_{N}\right)=\frac{1}{2} \sum_{\substack{i, j \\ i \neq j}}^{N} \frac{e^{2}}{\varepsilon\left|\mathbf{r}_{i}-\mathbf{r}_{j}\right|},
$$

где $\varepsilon-$ статическая диэлектрическая проницаемость, то гамильтониан системы запишется как

$$
\begin{aligned}
\hat{H}(1, \ldots, N)=\frac{1}{2 \mu} \sum_{j=1}^{N} \hat{P}_{j}^{2} & +\sum_{j=1}^{N} U_{\text {conf }}\left(\mathbf{r}_{j}\right) \\
& +V_{\text {int }}\left(\mathbf{r}_{1}, \ldots, \mathbf{r}_{N}\right) .
\end{aligned}
$$

Так как размерное квантование вдоль оси $z$ сильно, можно пренебрегать в данном направлении междырочным взаимодействием и рассматривать оператор взаимодействия между дырками $V_{\text {int }}$ как зависящий только от двумерных координат $\left\{\boldsymbol{\rho}_{1}, \boldsymbol{\rho}_{2}, \ldots, \boldsymbol{\rho}_{N}\right\}$ :

$$
\hat{V}_{\mathrm{int}} \equiv \hat{V}_{\mathrm{int}}\left(\boldsymbol{\rho}_{1}, \boldsymbol{\rho}_{2}, \ldots, \boldsymbol{\rho}_{N}\right)=\frac{1}{2} \sum_{\substack{i, j \\ i \neq j}}^{N} \frac{e^{2}}{\varepsilon\left|\boldsymbol{\rho}_{i}-\boldsymbol{\rho}_{j}\right|} .
$$

В рамках адиабатического приближения волновая функция системы запишется в виде произведения $[15,16]$ :

$$
\begin{aligned}
\Psi\left(\mathbf{r}_{1}, \ldots, \mathbf{r}_{N}\right)= & \chi_{n_{1}, \ldots, n_{N}}\left(z_{1}\left(\rho_{1}\right), \ldots, z_{n}\left(\rho_{N}\right)\right) \\
& \times F\left(\boldsymbol{\rho}_{1}, \ldots, \boldsymbol{\rho}_{N}\right),
\end{aligned}
$$

где через $\chi_{n_{1}, \ldots, n_{N}}\left(z_{1}\left(\rho_{1}\right), \ldots, z_{n}\left(\rho_{N}\right)\right)$ обозначено произведение одночастичных волновых функций $\chi_{n}(z)$,

$$
\chi_{n_{1}, \ldots, n_{N}}\left(z_{1}\left(\rho_{1}\right), \ldots, z_{n}\left(\rho_{N}\right)\right)=\prod_{j=1}^{N} \sqrt{\frac{2}{a\left(\rho_{i}\right)}} \sin \frac{\pi n_{j}}{a\left(\rho_{j}\right)} z_{j}
$$

Подставив (13) в уравнение Шредингера с гамильтонианом (11) и учитывая (14), после ряда преобразований $[15,16]$ можно показать, что выражение (11) сводится к виду

$$
\begin{aligned}
\hat{H}^{2 \mathrm{D}}= & \frac{1}{2 \mu} \sum_{j=1}^{N}\left(\hat{P}_{x j}^{2}+\hat{P}_{y j}^{2}\right) \\
& +\frac{\mu \Omega}{2} \sum_{j=1}^{N}\left(x_{j}^{2}+y_{j}^{2}\right)+\frac{1}{2} \sum_{\substack{i, j \\
i \neq j}} \frac{e^{2}}{\varepsilon\left|\boldsymbol{\rho}_{i}-\boldsymbol{\rho}_{j}\right|},
\end{aligned}
$$

где

$$
\Omega_{1}=\Omega_{2}=\ldots=\Omega_{N}=\frac{\pi \hbar}{\mu\left(h^{3} R\right)^{1 / 2}} .
$$

Далее, следуя [11], перейдем в (15) к новым переменным:

$$
\begin{gathered}
R=\sum_{j=1}^{N} \frac{\boldsymbol{\rho}_{j}}{\sqrt{N}}, \quad \mathbf{x}_{1}=\frac{\boldsymbol{\rho}_{1}-\boldsymbol{\rho}_{2}}{\sqrt{1 \cdot 2}}, \\
\mathbf{x}_{2}=\frac{\boldsymbol{\rho}_{1}-\boldsymbol{\rho}_{2}-2 \boldsymbol{\rho}_{3}}{\sqrt{2 \cdot 3}}, \ldots, \\
\mathbf{x}_{N-1}=\frac{\boldsymbol{\rho}_{1}+\boldsymbol{\rho}_{2}+\ldots-(N-1) \boldsymbol{\rho}_{N}}{\sqrt{(N-1) N}} .
\end{gathered}
$$

В результате гамильтониан $\hat{H}^{2 \mathrm{D}}$ представится в виде суммы двух независимых гамильтонианов: гамильтониана центра масс системы $\hat{H}_{\mathrm{CM}}^{2 \mathrm{D}}(\mathbf{R})$ и гамильтониана относительного движения дырок $\hat{H}_{\mathrm{Rel}}^{2 \mathrm{D}}\left(\mathbf{x}_{1}, \ldots, \mathbf{x}_{N-1}\right)$. При этом для $\hat{H}_{\mathrm{CM}}^{2 \mathrm{D}}$ имеем

$$
\hat{H}_{\mathrm{CM}}^{2 \mathrm{D}}(\mathbf{R})=-\frac{\hbar^{2}}{2 M}\left(d^{2} / d R^{2}\right)+M \Omega^{2} R^{2} / 2
$$

где $M$ суммарная масса дырок.

Пусть на рассматриваемую систему падает длинноволновое электромагнитное излучение с оператором возмущения

$$
\hat{H}^{\prime}=e \mathbf{E}(t) \sum_{j} \boldsymbol{\rho}_{j}=\sqrt{N} e \mathbf{E}(t) \mathbf{R},
$$

где $\mathbf{E}(t)=\mathbf{E}_{0} e^{-i \omega t}-$ вектор электрического поля падающего излучения.

Как следует из (19), $\hat{H}^{\prime}$ не содержит относительных координат $\left\{\mathbf{x}_{1}, \mathbf{x}_{2}, \ldots, \mathbf{x}_{N}\right\}$. Следовательно, под воздействием возмушения (19) в рассматриваемой системе будут иметь место переходы, аналогичные переходам в одночастичной системе с амплитудой электрического поля $\sqrt{N} E_{0}[11]$. Другими словами, в многочастичной системе реализуется одночастичный характер резонансных 
переходов, что является содержанием обобщенной теоремы Кона [11-13]. Теоретические оценки для энергии перехода дают значения

$$
\hbar \Omega_{\text {theor }}=\frac{\pi \hbar^{2}}{\mu\left(h^{3} R\right)^{1 / 2}} \approx 31 \mathrm{мэВ}
$$

для следующих параметров КТ, соответствующих наибольшим размерам точек:

$$
\begin{gathered}
h=2.95 \mathrm{Hм}, \quad \rho_{0}=9 \mathrm{Hм}, \\
R=\left(h^{2}+\rho_{0}^{2}\right) / 2 h=15.2 \mathrm{Hм}, \quad \mu=0.39 m_{0} .
\end{gathered}
$$

Экспериментально определенное значение равно: $\hbar \Omega_{\mathrm{ex}} \approx 30$ мэВ.

Таким образом, имеется хорошее совпадение теоретически вычисленных значений резонансных частот с экспериментально наблюдаемыми.

\section{5. Заключение}

Построенная на основе адиабатического приближения аналитическая теория размерного квантования дырок, связанных кулоновским взаимодействием, дает хорошее согласие с экспериментально определенными энергиями нижних внутризонных резонансов. При этом частоты резонансов не зависят от наличия взаимодействия и носят одночастичный характер, что происходит за счет специфической геометрии КТ, благодаря чему в рассматриваемой системе реализуются условия выполнения обобщенной теоремы Кона.

Работа выполнена при финансовой поддержке Министерства образования и науки России (государственные задания № 3.933.2017/4.6 и 3.6153.2017/7.8), грантом президента Российской Федерации для молодых кандидатов наук МК-6064.2016.2 и программой базового финансирования ГКН Республики Армения „Исследование физических свойств наноструктур со сложной геометрией и различными ограничивающими потенциалами“.

\section{Список литературы}

[1] D.N. Lobanov, A.V. Novikov, K.E. Kudryavtsev, A.N. Yablonskiy, A.V. Antonov, Yu.N. Drozdov, D.V. Shengurov, V.B. Shmagin, Z.F. Krasilnik, N.D. Zakharov, P. Werner. Physica E, 41, 935 (2009).

[2] Z.F. Krasilnik, A.V. Novikov, D.N. Lobanov, K.E. Kudryavtsev, A.V. Antonov, S.V. Obolenskiy, N.D. Zakharov, P. Werner. Semicond. Sci. Technol., 26, 014029 (2011).

[3] M. Grydlik, F. Hackl, H. Groiss, M. Glaser, A. Halilovic, T. Fromherz, W. Jantsch, F. Schaffler, M. Brehm. ACS Photonics, 3, 298 (2016).

[4] A.I. Yakimov, V.V. Kirienko, V.A. Armbrister, A.A. Bloshkin, A.V. Dvurechenskii. Appl. Phys. Lett., 107, 213502 (2015).

[5] A.I. Yakimov, V.V. Kirienko, A.A. Bloshkin, V.A. Armbrister, P.A. Kuchinskaya, A.V. Dvurechenskii. Appl. Phys. Lett., 106, 032104 (2015).
[6] M.E1 Kurdi, S. Sauvage, G. Fishman, P. Boucaud. Phys. Rev. B, 73, 195327 (2006).

[7] A. Tonkikh, N. Zakharov, V. Talalaev, P. Werner. Phys. Status Solidi RRL, 4 (8-9), 224 (2010).

[8] A. Tonkikh, P. Werner. Phys. Status Solidi B, 250 (9), 1795 (2013).

[9] A. Tonkikh, N. Zakharov, E. Pippel, P. Werner. Thin Sol. Films, 519 (11), 3669 (2011).

[10] A.N. Sofronov, L.E. Vorobjev, D.A. Firsov, R.M. Balagula, A.A. Tonkikh. Superlatt. Microstruct., 107, 228 (2017).

[11] А.О. Говоров, А.В. Чаплик. Письма ЖЭТФ, 52, 31 (1990).

[12] P.A. Maksym, T. Chakraborty. Phys. Rev. Lett., 65, 108 (1990).

[13] F.M. Peeters. Phys. Rev. B, 42, 1486 (1990).

[14] W. Kohn. Phys. Rev., 123, 1242 (1961).

[15] D.B. Hayrapetyan, E.M. Kazaryan, H.A. Sarkisyan. J. Contemp. Phys., 48 (1), 32 (2013).

[16] D.B. Hayrapetyan, E.M. Kazaryan, H.A. Sarkisyan. Physica E, 75, 353 (2016).

[17] В.М. Галицкий, Б.М. Карнаков, В.И. Коган. Задачи по квантовой механике (М., Наука, 1992) гл. 8, с. 96.

[18] L.S. Petrosyan. Proc. NAS RA — Physics, 40 (4), 265 (2005).

Редактор Л.В. Шаронова

\section{Absorption of far infrared radiation in $\mathrm{Ge} / \mathrm{Si}$ quantum dots}

A.N. Sofronov ${ }^{1}$, R.M. Balagula ${ }^{1}$, D.A. Firsov ${ }^{1}$, L.E. Vorobjev' ${ }^{1}$, A.A. Tonkikh ${ }^{2}$, H.A. Sarkisyan ${ }^{1,3}$, D.B. Hayrapetyan ${ }^{3}$, L.S. Petrosyan ${ }^{3,4}$, E.M. Kazaryan ${ }^{3}$

${ }^{1}$ Peter the Great Saint Petersburg

Polytechnic University,

195251 St. Petersburg, Russia

2 OSRAM Opto Semiconductors,

Regensburg, Germany

${ }^{3}$ Russian-Armenian University,

Yerevan, Armenia

${ }^{4}$ Jackson State University,

USA

Abstract The experimental and theoretical studies of the optical absorption in doped Ge/Si quantum dot structures in the far IR spectral range are described in this paper. The far IR spectral range corresponds to the energies of the optical transitions from the quantum dot ground state to the lowest excited state. Under the adiabatic approximation we built the analytical theory of the spatial confinement of holes in a lens-shaped quantum dot taking into account the paired Coulomb interaction. We show that the frequencies of the lowest inter-level resonances are not influenced by the presence of the hole-hole interaction. This fact corresponds to the generalized Kohn's theorem that is fulfilled due to the specific dot geometry. Experimental and theoretical values of the transition energies are in a good agreement. 